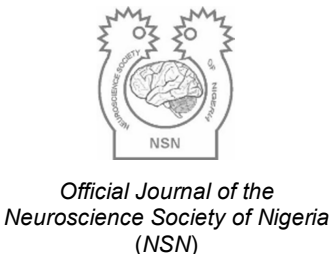

ORIGINAL ARTICLE

\title{
Evaluation of the Neuroprotective Effects of Eugenol on Formaldehyde Induced Neurotoxicity in Wistar Rats
}

\author{
Samuel B. Mesole ${ }^{1}$, Omachonu A. Okpanachi ${ }^{2}$, Sunday S. Adebisi ${ }^{3}$, Uthman A.Yusuf ${ }^{4}$, \\ Elvis T. Godam ${ }^{5}$, Chanda G. Chisunka ${ }^{6}$, Andrew Ivang ${ }^{7}$, Animoku A. Abdulrazaq ${ }^{8}$, \\ and Tosin J. Kolawole ${ }^{3}$
}

\begin{abstract}
${ }^{1}$ Department of Human Anatomy, School of Medicine, Texila American University, Zambia; ${ }^{2}$ Department of Physiology, Faculty of Biomedical Sciences, Kampala International University, Uganda; ${ }^{3}$ Department of Human Anatomy, Faculty of Basic Medical Sciences, Ahmadu Bello University, Zaria, Nigeria; ${ }^{4}$ Department of Anatomy, Mulungushi University, Zambia; ${ }^{5}$ Department of Human Anatomy, Rivers State University, Port Harcourt, Nigeria; ${ }^{6}$ Department of Human Physiology, Texila American University, Zambia; ${ }^{7}$ Clinical Anatomy Unit, Department of Clinical Biology, University of Rwanda; ${ }^{8}$ Department of Anatomy, Kogi State University, Ayingba, Nigeria
\end{abstract}

Received:

June 2020

Accepted:

September 2020

\begin{abstract}
Over the years, Formaldehyde (FA) has been linked to increased generation of reactive oxygen species (ROS), leading to oxidative stress and cognitive decline. However limited numbers of studies have shown the effect of eugenol on FA induced toxicity in Wistar rats. Therefore this study aimed at investigating the effects of eugenol on the FA induced toxicity in Wistar rats. A total of twenty male Wistar rats where divided into four groups: (Group I. $150 \mathrm{mg} / \mathrm{kg}$ eugenol; Group II, $5 \mathrm{mg} / \mathrm{kg}$ FA; Group III, $150 \mathrm{mg} / \mathrm{kg}$ eugenol $+5 \mathrm{mg} / \mathrm{kg} \mathrm{FA}$; Group IV/control, $2 \mathrm{ml} / \mathrm{kg}$ distilled water) for thirty days. FA and eugenol were administered orally. Rats were humanely sacrificed under $0.8 \mathrm{mg} / \mathrm{kg}$ ketamine anaesthesia administered intraperitoneally. Cognitive tests using Morris water maze and Novel object recognition test were carried out, Oxidative stress parameters, acetylcholine activity and histometric analysis of hippocampal Cornu Ammonis (CA 1 and 3) pyramidal neuronal cells. Administration of FA resulted in significant $(p<0.05)$ increased activity of malondialdehyde (MDA), intra-mitochondrial accumulation of 8-hydroxydeoxyguanosine (8-OHdG), reduced activity level of superoxide dismutase (SOD) and acetylcholine levels. However co-administration of eugenol and FA resulted in significant $(p<0.05)$ enhancement of cognitive ability and also significantly $(p<0.05)$ reduced MDA and $8-O H d G$ levels, and increased SOD and acetylcholine levels. Our results indicate that eugenol would provide therapeutic value against FA induced oxidative stress and cognitive impairments.
\end{abstract}

Key words: 8-hydroxydeoxyguanosine; Histometry; Superoxide dismutase; Malondialdehyde; Hippocampus

\section{INTRODUCTION}

Formaldehyde (FA) is a colourless, highly water soluble aldehyde, produced naturally as a by-product of cellular metabolism. Its ingestion occurs majorly via topical or oral routes. The highest level of ingestion is typically through the respiratory system. FA ingestion into biological systems is usually in the form of fumes which are produced during the combustion process of fossil fuels, cigarettes and paints (Nazaroff and Singer 2004). Other routes by which it can be ingested include fresh water, food and drugs. In food, FA can occur naturally or as a contaminant used as a preservative or disinfectant. It can also result from cooking or smoking of foods (Yang et al. 2007). Infants can be exposed through

Correspondence: Samuel B. Mesole, MSc, Department of Human Anatomy, School of Medicine, Texila American University, Zambia; ms361450@gmail.com; +2348035449746 
vaccines present in diphtheria, polio and tetanus (Thaysen-Andersen et al. 2007). Several drugs targeted at malignant tumours are formulated with FA (required for drug activation) (Evison et al. 2008). Some hair treatment products which requires heat produces FA vapour increasing the stylist exposure to FA. Some students who study anatomy and dissect formalin embalmed cadavers are exposed to FA vapour which results in itchy skin, blocked sinuses and other forms of respiratory tract discomfort. Within the limbic system, the hippocampus performs a vital role in learning and memory (Morris et al. 1982). Studies showed that inhaled FA exposure has a detrimental effect on the ability of spatial learning and memory in rats (Pitten et al. 2000; Malek et al. 2003; Ahmed et al. 2007; Lu et al. 2008; Liu et al. 2010). These suggests that FA has deleterious effects on various hippocampal functions.

Formaldehyde is known to induce oxidative stress, cognitive deficits and produces imbalance between pro-apopotic and anti-apoptotic proteins, thereby triggering apoptosis. Eugenol as a naturally occurring component of plants such as clove, Occimum gratissimum and Ocimum sanctum. It is an established antioxidant that helps re-establish balance between pro- and anti-apoptotic proteins induced by aluminium chloride (Mesole et al. 2020).

The study therefore investigated the neuroprotective effect of eugenol on FA induced toxicity, aimed at obtaining an alternative therapeutic potential with minimal side effects.

\section{MATERIALS AND METHODS}

Twenty adult male Wistar rats with average weight of 180-200 g, and obtained from the animal house of Texila American University, Zambia were allowed free access to food and water. This research was approved by the Texila American University Research and Ethics Committee. Experiments were carried out in accordance with the National Institute of Health Guide for the Care and Use of Laboratory Animals (Institute of Laboratory Animal Resources Committee, 2011). All measures were put in place to significantly reduce animal suffering. Wistar rats where divided into four groups as follows: Group I (EG) was given $150 \mathrm{mg} / \mathrm{kg}$ eugenol (Mesole et al. 2020); Group II (FA) was given 5 mg/kg FA (Hegazy et al. 2017); Group III (EG+FA) was given $150 \mathrm{mg} / \mathrm{kg}$ eugenol + $5 \mathrm{mg} / \mathrm{kg} \mathrm{FA}$; and Group IV (CTRL) was the Control.

Rats were weighed at the beginning of the experiment and $24 \mathrm{~h}$ after last administration. FA was administered intraperitoneally, while eugenol was administered orally throughout the duration of this study (30 days). At the end of the study ( $24 \mathrm{~h}$ after last administration, day 31) animals where sacrificed humanely using ketamine as anaesthesia and injected intraperitoneally at a dose of $0.8 \mathrm{mg} / \mathrm{kg}$ (Mesole et al. 2020).

The rat brains were carefully removed and stripped of any adhering structures, washed twice in ice cold 50 $\mathrm{mM}$ Tris $-\mathrm{HCl}$ at $\mathrm{pH}$ 7.4. The rat brains were weighed using a sensitive digital weighing balance and the left cerebral hemisphere was homogenized to yield a $10 \%(w / v)$ homogenate in ice-cold medium. These contained $50 \mathrm{mM}$ Tris $-\mathrm{HCl}$ at $\mathrm{pH}$ 7.4. The homogenates obtained was centrifuged at $1200 \mathrm{~g}$ for $10 \mathrm{~min}(600 \mathrm{sec})$ at $4{ }^{\circ} \mathrm{C}$. The supernatants obtained, was used for various protein and enzymatic estimations.

FA (\#50-00-0) was obtained from Sigma Chemical Co (St. Louis, MO) and was dissolved in phosphate buffered saline (PBS). Eugenol (\#58-23-4; Yueyang Jiazhiyuan Biological Co Ltd, Hunan China). Malondialdehyde (MDA), superoxide dismutase (SOD), 8-OHdG and acetylcholine enzyme linked immunosorbent assay (ELISA) kits where obtained from Elabscience Biotechnology Inc.

\section{Markers of Oxidative Stress \\ Malondialdehyde and Superoxide Dismutase}

MDA, which is generated as a by-product of lipid peroxidation (LPO), was determined as an indicator of LPO (Gutteridge, 1995; DeZwart et al. 1999). Thiobarbituric acid (TBA) reagent which contains $15 \%$ trichloroacetic acid, $0.375 \%$ TBA and $0.25 \% \mathrm{M}$ $\mathrm{HCl}$ reacted with MDA, which was derived from polysaturated fatty acids during peroxidation.

The anti-oxidative enzyme, SOD activity was measured spectrophotometrically as an inhibitor of epinephrine spontaneous auto-oxidation at $480 \mathrm{~nm}$. To assay the enzyme activity, carbonate buffer (50 $\mathrm{mM}, \mathrm{pH}$ 10.2, which contained $0.1 \mathrm{mM}$ EDTA, was added to the sample and afterwards, $10 \mathrm{mM}$ epinephrine was also added. (Nishigori et al. 1989). Data were expressed as SOD units/mg protein. The amount of cellular protein was determined according to the method of (Lowry et al. 1951). Bovine serum albumin served as standard.

\section{Oxidative Stress in Mitochondrial DNA}

Intra-mitochondrial accumulation of (8-hydroxyde oxyguanosine) 8-OHdG was measured as an indicator of mitochondrial DNA (mtDNA) oxidative damage using highly sensitive 8-OHdG Check ELISA Kits. Mitochondrial DNA was isolated using the method of Mecocci et al. (1994) and reported by Ding and Yang (2010). Each left cerebral hemisphere was homogenized in buffer $(\mathrm{pH} 7.5)$ containing $0.21 \mathrm{M}$ mannitol, $0.07 \mathrm{M}$ sucrose, $0.05 \mathrm{M}$ Tris- $\mathrm{HCl}, 0.2 \mathrm{M}$ ethylenediaminetetraacetic acid (EDTA) and $3 \mathrm{mM}$ $\mathrm{CaCl}_{2}$. A centrifugation at $1500 \times \mathrm{g}$ for $15 \mathrm{~min}$ was performed. The supernatant was collected and centrifuged at $1500 \times \mathrm{g}$ for $10 \mathrm{~min}$ again, and then the resulting supernatant was again centrifuged at $14,000 \times \mathrm{g}$ for $20 \mathrm{~min}$. The deposit was collected and 
re-suspended in Tris-EDTA buffer ( $\mathrm{pH}$ 7.4). Sodium dodecyl sulphate (SDS) (2\%) and $400 \mu \mathrm{g} / \mathrm{ml}$ proteinase $\mathrm{K}$ were added to lyse the cells. Samples were incubated at $37{ }^{\circ} \mathrm{C}$ for $4 \mathrm{~h}$. After three extractions with high purity distilled phenol and chloroform-isoamyl alcohol, mtDNA was precipitated with $1 / 10$ volume of $3 \mathrm{M}$ sodium acetate $(\mathrm{pH} 7.4)$ and 2 volumes of ethanol at $-20{ }^{\circ} \mathrm{C}$ overnight. Isolated mtDNA was digested with DNase I (200 U/mg DNA). Nuclease P1 and alkaline phosphatase $(10 \mathrm{U} / \mathrm{mg}$ DNA) in the presence of $40 \mathrm{mM}$ Tris- $\mathrm{HCl}(\mathrm{pH}$ 8.5) containing $10 \mathrm{mM} \mathrm{MgCl}_{2}$. Samples were incubated for $4 \mathrm{~h}$ at $37^{\circ} \mathrm{C}$, then the kits were used to determine the level of 8-OHdG.

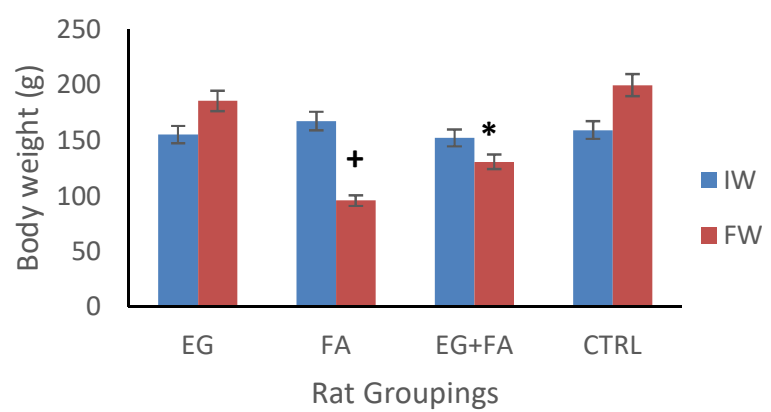

Fig. 1: The effect of eugenol on body weight of Wistar rat following administration of FA. $n=5$; mean \pm SEM. Turkey's post-hoc test: ${ }^{*}=p<0.05$ when compared with $\mathrm{FA} ;+=p<0.05$ when compared with control group. Initial weight (IW), Final weight (FW). Control (CTRL), Eugenol (EG), Formaldehyde (FA)

\section{Acetylcholine Assay Protocol}

Acetylcholine activity was assayed using brain homogenates and following the protocol as used by (Miranda and Bermúdez-Rattoni 1997) and using the appropriate ELISA kit.

\section{Behavioural Studies}

The following behavioural tests were conducted during the course of this study:

\section{Novel Object Recognition Test}

The object recognition test is now among the most commonly used behavioural tests for rats. A rat is presented with two similar objects during the first session, and then one of the two objects is replaced by a new object during a second session. The amount of time taken to explore the new object provides an index of recognition memory (Leger et al. 2013). This test was carried out $24 \mathrm{~h}$ before commencement of treatment (pre-treatment), day 7 , 15 and 30 at $06: 00 \mathrm{~h}$ (6:00 am local time).

This method as described by Brown et al. (1999) and Podhorna and Brown (2004) was used. Prior to testing all rats were habituated to the open field arena for $5 \mathrm{~min}$. The rats were carried to the test room in their home cages and run individually. After each 5 min trial, the rat was returned to their home cages and the apparatus cleaned with $70 \%$ ethyl alcohol and permitted to dry between trials. Two objects $A$ and $B$ (which were similar) were placed in diagonal corners opposite each other. Each rat was allowed to explore the arena. After 15 min intertrial interval, Object $C$ (novel object) was introduced as the new object replacing either object A or B. During the test, behaviour scored was the time taken (sec) by rat to explore new object C. $24 \mathrm{~h}$ before commencement of treatment, time taken to explore object $\mathrm{C}$ was designated as pre-treatment.

\section{Morris Water Maze}

The method described by Morris et al. (1982), and reported by Drapeau et al. (2003) for spatial memory and learning was adopted for this study. Rats were tested in a Morris water maze $(180 \mathrm{~cm}$ diameter, 60 $\mathrm{cm}$ height) filled with water. An escape platform was hidden $2 \mathrm{~cm}$ below the surface of the water in a fixed location in one of the four quadrants halfway between the wall and the middle of the pool. This test was carried out at 17:00 h (5:00 pm local time) and $24 \mathrm{~h}$ before the commencement of treatment was designated as pre-treatment, while further test was carried out on day 7,15 and 30 respectively.

Before the commencement of the treatment, the rats were initially trained in the pool daily for four days. During the training, animals were required to locate the submerged platform by using distal extra-maze cues. They were tested for four trials per day (90 sec with an inter trial interval of $30 \mathrm{sec}$ and beginning from different start points that varied randomly each day). When the animal did not find the platform, the animal was guided in locate the platform. The time taken to find the hidden platform was recorded as latency (sec).

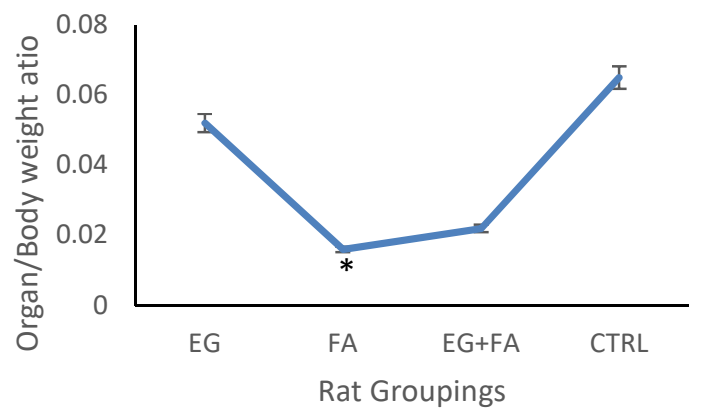

Fig. 2: Effect of eugenol on organ/bogy weight ratio following administration of $F A$. $n=5$; mean \pm SEM; Turkey's post-hoc test: $*=p<0.05$ compared to the control. Control (CTRL), Eugenol (EG), Formaldehyde (FA)

\section{Morphometric Studies}

Brains were removed and cleaned of any adhering structures, weighed and the organ/body weight ratio was computed. Brain tissues were fixed in Bouin's 
fluid (right cerebral hemisphere was used for morphometric studies while the left was used for all biochemical assays), embedded in paraffin sections and sectioned at a thickness of $5 \mu \mathrm{m}$, and stained with cresyl fast violet. Cresyl fast violet micrographs (digital microscopic images) were used for histometry. Three micrographs were randomly captured (Kaizaki et al. 2003; Lai et al 2011) with emphasis on the hippocampal Cornu Ammonis (CA 1 and 3 ) cells. Five to twenty cells were randomly selected, measured and averaged in three micrographs (Andersen and Pakkenberg, 2003; Jelsing et al. 2006).

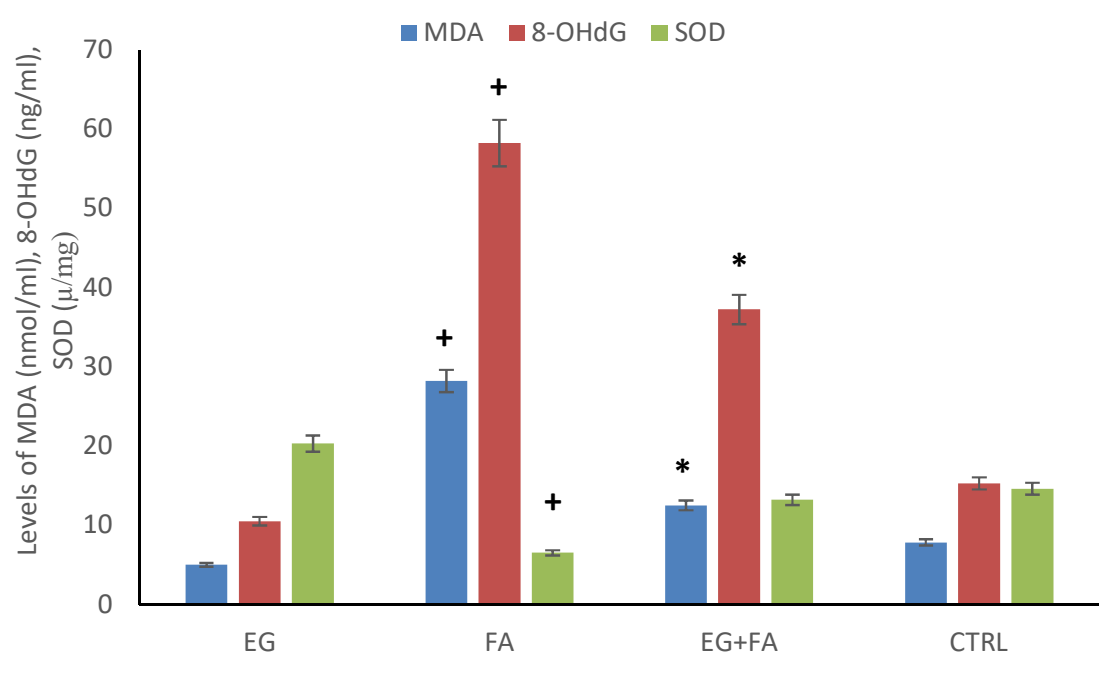

Fig. 3: Effect of eugenol on brain levels of MDA, 8-OHdG and SOD following the administration of FA. $n=5$; mean \pm SEMI Turkey's post-hoc test. * $=p<0.05$ compared with FA group; $+=p<0.05$ compared with control group. Malondialdehyde (MDA), 8-hydroxydeoxyguanosine (8-OHdG), superoxide dismutase (SOD), Control (CTRL), Eugenol (EG), Formaldehyde (FA)

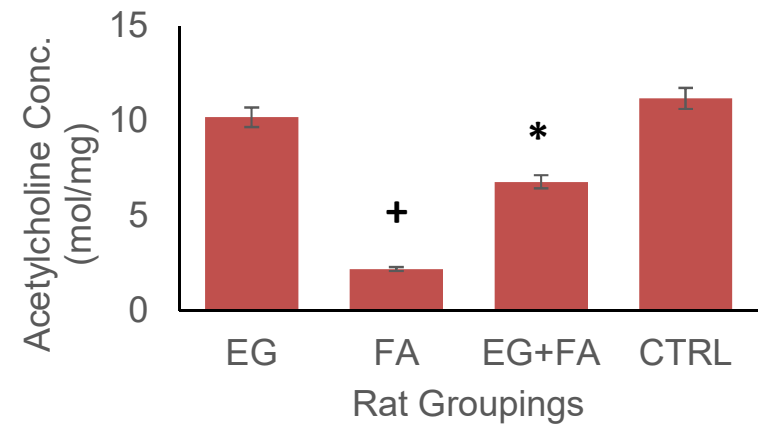

Fig. 4: The effect of eugenol on the brain levels of acetylcholine following administration of FA. $\mathrm{n}=5$; mean \pm SEM One way Turkey's post-hoc test: ${ }^{*}=p<0.05$ compared with FA treated group; $+=p<0.05$ compared with the control group. Control (CTRL), Eugenol (EG), Formaldehyde (FA)
This is briefly described below:

Using a light microscope with $\times 25$ objective $(\times 250$ magnification) -and computer running image processing software (Amscope MT version 3.0.0. 5, USA) for microscopy, digital microscopic images were captured. Histometry and calibration (using the Amscope Microscope Cameras; Psi Wave function, 2009). Firstly a micrometre slide (microscope slide with scale on it), it is divided $10 x$ into $100 \mu \mathrm{m}$ units and is captured at the same magnification and quality (pixels) the micrograph was photographed. A line tool was selected and this was used to carefully measure from start of one major line (of the captured microscope slide) to the start of the next, or end to end. Recorded length in pixels; in this study was 408 pixels $/ 100 \mu \mathrm{m}$ or $4.08 \mathrm{pixel} / \mu \mathrm{m}$. A polygon tool was used to measure the area and perimeter of desired cells. Values are computed and presented automatically by the software.

Statistical Analysis
Results obtained from this research were analysed with Statistical Package for Social Sciences (IBM SPSS version 21.0, SPSS Inc.) and Microsoft office Excel 2007 for charts. These results were expressed as mean \pm standard error of mean (SEM) and significant differences among means of the groups was determined with one way analysis of variance (ANOVA) with Turkey's post-hoc test for significance. Paired sample t-test was used for comparison of mean. Values were considered significant when $\mathrm{p} \leq 0.05$.

\section{RESULTS}

\section{Body Weight}

Figure 1 shows the effect of eugenol on body weight of Wistar rat following administration of $F A$. This result showed a significant $(p<0.05)$ reduction in the final weight (FW) of the group treated with FA when compared with the control. Treatment with eugenol $(E G+F A)$ significantly $(p<0.05)$ preserved the body weight when compared to the FA treated group

\section{Organ/Body Weight Ratio}

Figure 2 shows the effect of eugenol on brain/body weight of rats following administration of $F A$. This result showed a significant $(p<0.05)$ reduction in the organ/body weight ratio of the FA treated group. 
However treatment with eugenol $(E G+F A)$ resulted in a non-significant $(p>0.05)$ change in organ/body weight ratio.

\section{Markers of Oxidative Stress and Biochemical Parameters}

Figure 3 shows the effect of eugenol on tissue activity of MDA, 8-OHdG and SOD following administration of FA. This result showed a significant $(p<0.05)$ increase in the brain activity of MDA and 8-OHdG upon administration of FA. Co-administration of eugenol and $F A(F A+E G)$ resulted in a significant $(p<0.05)$ decrease in the brain activity of MDA and 8$\mathrm{OHdG}$. Administration of eugenol only (EG) resulted in a non-significant reduction in tissue levels of MDA and $8-\mathrm{OHdG}$ when compared to the control group. There was a significant $(p<0.05)$ decrease in the tissue levels of SOD within the group administered FA when compared to the control group. Coadministration of eugenol and $F A(F A+E G)$ resulted in a non- significant $(p>0.05)$ increase in tissue levels of SOD when compared to the group administered FA only. In the group administered eugenol only (EG) there was a non-significant $(p>0.05)$ increase in brain tissue levels of SOD.

Figure 4 shows the effect of eugenol on the levels of acetylcholine following the administration of FA. This results revealed a significant $(p<0.05)$ reduction in the brain levels of acetylcholine in the group treated with FA when compared to control. However treatment with eugenol resulted in a significant $(p<0.05)$ elevation of acetylcholine when compared to the group treated with FA only.

\section{Behavioural Study Novel Object Recognition Test}

Figure 5 shows the effect of eugenol on cognition (novel object recognition) following administration of FA. The result revealed a significant $(p<0.05)$ reduction in cognition in the group treated with $F A$ when pre-treatment is compared to day 30, this reduction was found to be significant $(p<0.05)$ when compared to the control. Animals administered eugenol only revealed a significant $(p<0.05)$ increased cognitive ability when pre-treatment is compared to day 30. However treatment with eugenol $(E G+F A)$ revealed a non-significant $(p>0.05)$ reduction when pre-treatment is compared to Day 30 but when compared to control on day 30 there was a significant $(p<0.05)$ difference.

\section{Morris Water Maze}

Figure 6 shows Morris water maze test, a well validated test for spatial learning and memory used to assess learning and memory in experimental animals. Performance in the training/habituation sessions revealed reduced latency (time) to find the hidden platform between day 3 and 4 of the training sessions

\section{Histometry}

Figure 8 shows the effect of eugenol on area $\left(\mu \mathrm{m}^{2}\right)$ of hippocampal (CA1 and CA3) pyramidal cell soma following administration of FA. This result revealed a

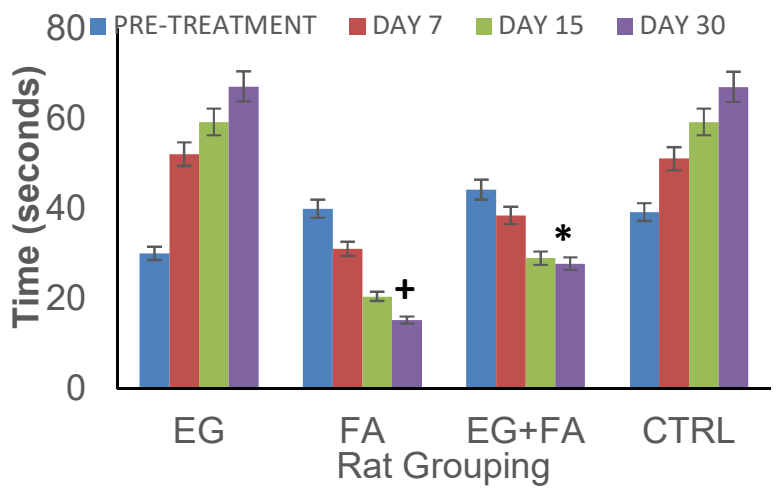

Fig. 5: Effect of eugenol on cognition (novel object recognition) following treatment with $F A . n=5$; mean \pm SEM; Turkey's post-hoc test $+=p<0.05$ compared to control group on day $30 ;{ }^{*}=p<0.05$ compared to FA group on day 30. Control (CTRL), Eugenol (EG), Formaldehyde (FA)

significant $(p<0.05)$ reduction the area of pyramidal neurons in the CA1 and CA3 region of FA treated group when compared to the control. Treatment with eugenol resulted in a significant $(p<0.05)$ increase in the area of hippocampal neurons when compared to the FA treated group.

\section{DISCUSSION}

Adult Wistar rats were used for the entire duration of this study, since they are easier to be housed, bred and handled easily. Intraperitoneal administration of FA resulted in significant reduction in body weight, indicative of interference with animal's metabolism and this was also reported by Til et al. (1988).

$\mathrm{FA}$ is a major air pollutant and an important chemical with a wide variety of usage. In this study, we investigated the role of FA generating free radicals

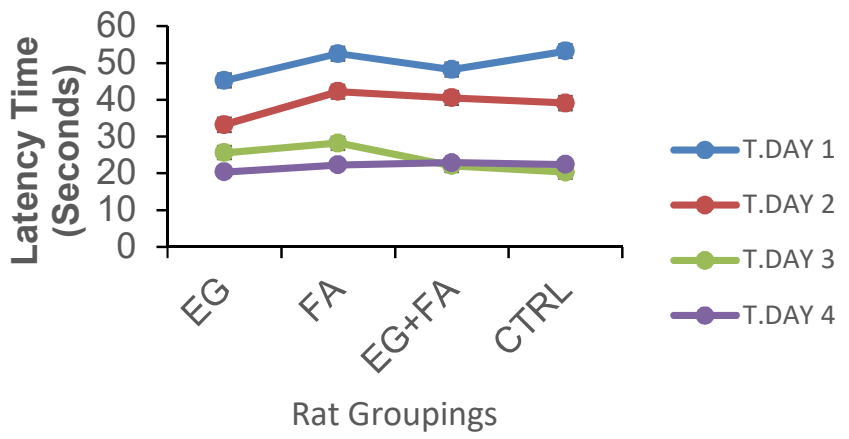

Fig. 6: Transfer latency of Wistar rats in Morris water maze habituation. Control (CTRL), Eugenol (EG), Formaldehyde (FA) 
which will result in oxidative stress (we measured the levels SOD and MDA), activity levels of acetylcholine and role of FA in learning and memory (Morris water maze and novel object recognition test). We demonstrated that intraperitoneal administration of FA can result to: (1) Diminished learning and memory; (2) Generation of free radicals; (3) Accumulation of $8-\mathrm{OHdG}$ within the mitochondria; and (4) Diminished levels of acetylcholine. The results have shown FA has the capacity induce oxidative stress. We measured the levels of MBA and SOD. MDA is produced from lipid peroxidation and can be used to assess the oxidative damage of lipids (Janero 1990). This implies that the higher the level of MDA in tissue, the higher the level of oxidation. In our study, a high level of tissue MDA was observed among animals treated with FA as reported by Lu et al. (2008). However, treatment with eugenol was able to reduce the tissue levels of MDA among treated animals as reported by Mesole et al (2020). SOD plays vital roles in anti-oxidation within organs and tissues (Kurata et al. 1993). The activity levels of SOD would therefore infer or indicate anti-oxidation within biological systems. In our study, intraperitoneal administration of FA resulted in diminished activity levels of SOD; however, treatment with eugenol resulted in elevated levels of tissue SOD.

Oxidative stress is a disturbance between prooxidant and antioxidant balance favouring prooxidant, increasing the potential to cause damage (Sies 1991). The brain requires and consumes a large amount of oxygen, these makes it particularly highly susceptible to oxidative stress (Andersen 2004). Reactive oxygen species (ROS) are excessively produced in oxidative stress, reversibly or irreversibly damage compounds of all biochemical classes. These include nucleic acids, proteins, free amino acids, lipids and lipoproteins, carbohydrates etc. ROS can impair cell activities such as membrane function and gene expression. If not removed by natural scavengers, damage within the tissue/system occurs through peroxidation of structurally vital polyunsaturated fatty acids within membrane phospholipid (Lu et al. 2008).

The mitochondrion is a major producer of reactive oxygen species in mammalian cells. Close proximity to reactive oxygen species makes the mitochondria DNA (mtDNA) susceptible to oxidative damage (Shigenaga et al. 1994). Studies have revealed that 8-hydroxy-2' -deoxyguanosine (8-OHdG) is a marker of ROS induced damage to mtDNA (Hang et al.
2011; Mahmoud and Marwa 2017; Mesole et al. 2020). In this study, administration of FA resulted in increased intra-mitochondrial accumulation of 8-

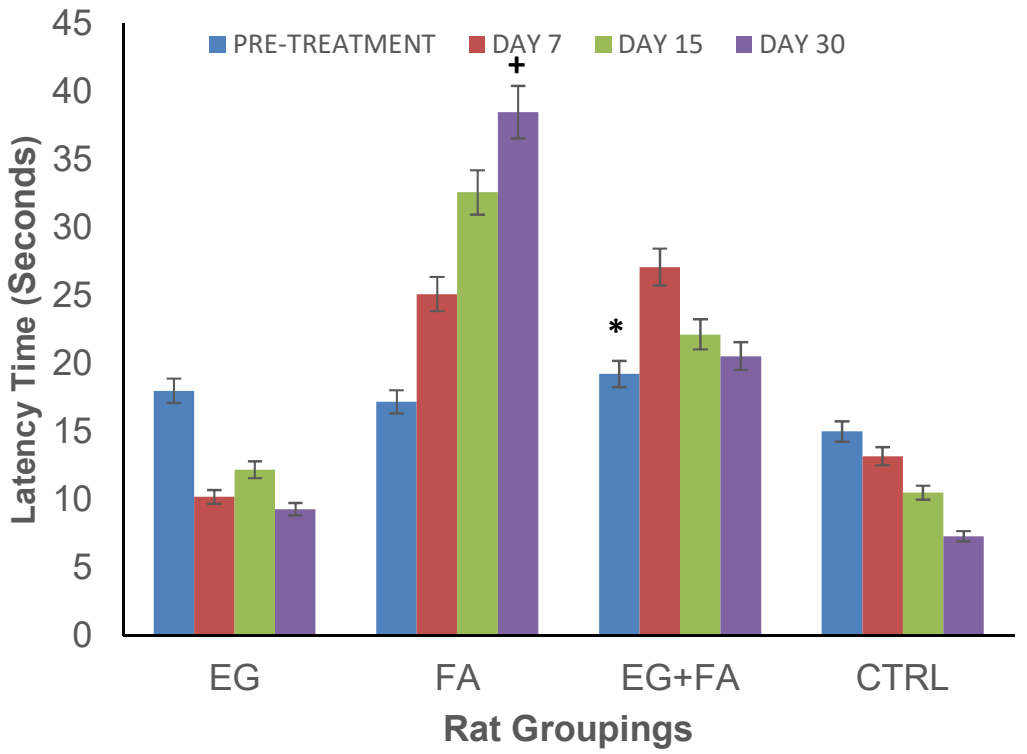

\section{Rat Groupings}

Fig. 7 shows the effect of eugenol on cognition (Morris water maze) following administration of FA. $n=5$; mean \pm SEM One way Turkey's post-hoc test ${ }^{*}=p<0.05$ compared to FA on day $30 ;{ }^{*}=p<0.05$ compared to FA on day $30 ;+=p<0.05$ compared to the control on day 30 . Control (CTRL), Eugenol (EG), Formaldehyde (FA)

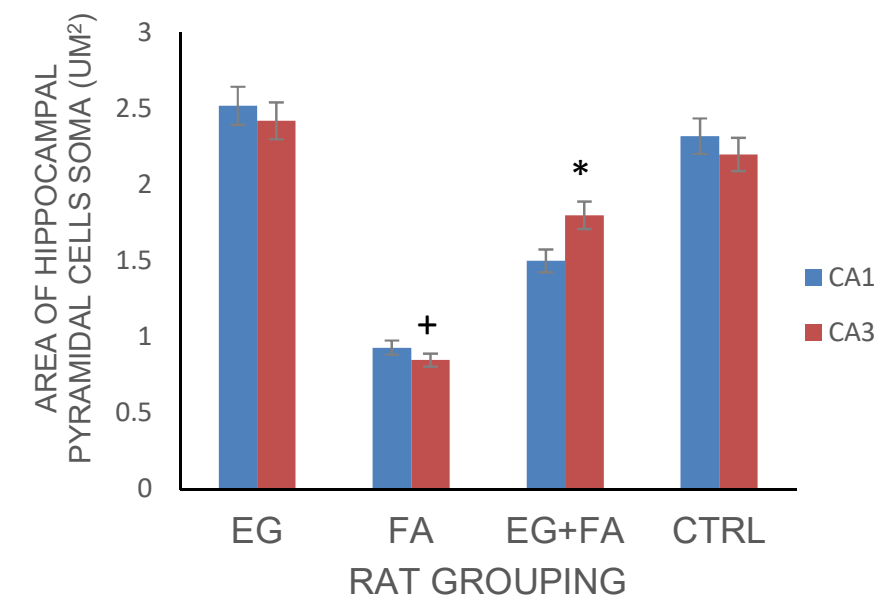

Fig. 8: Effect of eugenol on the area of pyramidal neuronal cells of the hippocampus (CA1 and CA3) following administration of FA. $n=5$; mean \pm SEM One way Turkey's post-hoc test $+=p<0.05$ compared to the control group. ${ }^{*}=p<0.05$ compared to the FA treated group. Control (CTRL), Eugenol (EG), Formaldehyde (FA)

OHdG, and administration of eugenol resulted in reduced levels of 8-OHdG as reported by Mahmoud and Marwa (2017) and Mesole et al. (2020). Accumulation of 8-OHdG has also been linked with cognitive decline in a previous study (Gao et al. 2010). 
Acetylcholine activity level has been linked to memory and learning, and diminished levels of acetylcholine has been linked to impairment of cognitive abilities (Ridley et al. 1984). Exposure to FA at higher levels has been linked to diminished activity levels of acetylcholine as reported previously (David et al. 2000; Zendehdel et al. 2016). In this study, intraperitoneal administration of FA resulted to diminished activity levels of acetylcholine, and this can be linked to the poor performance in the Morris water maze and novel object recognition tasks. However treatment with eugenol resulted in increased level of acetylcholine.

Acetylcholine plays an important function in enhancing learning and memory processes within the hippocampus. Activity of acetylcholine in the hippocampus was linked with memory. Hence restoration of acetylcholine levels in the hippocampus is sufficient to rescue diminished memory and learning. Acetylcholine plays a vital role in encoding new memories, neural mechanism of memory from working memory to long-term memory, promotion of memory formation, consolidation, retrieval and enhancement hippocampal and synaptic plasticity.

Studies involving learning and memory play a vital role in the study of neurological disorders that has cognitive components, example of such are schizophrenia and Alzheimer's disease. The most widely used spatial navigation task is the Morris water maze (Morris 1984; Brandies et al. 1989),

In this study, cognition was examined (learning and memory) using Morris water maze and novel object recognition task. These tests were carried out on day 7, 15 and 30. The FA treated group had an increased latency from day 7 to 30 , but treatment with eugenol reduced the latency, indicative of improved cognition. The novel object recognition task also used to test for cognition showed a reduction in time spent exploring the novel (new) object, indicative of impaired cognitive function. Administration of eugenol however lead to an increase in the time spent on novel object, indicative of enhanced cognitive ability which was also previously reported (Liu et al. 2013).

Histometry is the measurement of the dimensions of cellular component of tissues. Histologic sections are used to define the normal or abnormal appearance of the brain tissue (Anderson 1989). Histometry provides a sound basis for comparison of histological observation. It offers objectivity, increases precision compared with direct visual appraisal and makes statistical analysis easier. It improves assessment of certain histological change, which though may be recognizable by the eye, are accurately graded and their progression better appreciated by histometric quantization (Huda and Zaid 2007; Ahmed et al. 2018). In this study, histometric analysis of pyramidal cells in the CA1 and CA3 hippocampal region was carried out. Treatment with FA showed reduction in area of pyramidal neuronal cells, indicative of toxicity induced treatment and might result in neuronal death and hence poor cognitive performance in cognitive test and reduced acetylcholine levels in the FA treated groups. However treatment with eugenol was able to preserve pyramidal neuronal area close to the control group.

\section{Conclusion}

In summary, exposure to FA can result in generation of free radicals, which resulted in elevation of MDA, intra-mitochondrial accumulation of $8-\mathrm{OHdG}$, reduced tissue activity of acetylcholine, SOD and reduction in cognitive ability of Wistar rats. However administration of eugenol resulted in improved cognitive performance and increased tissue activity of acetylcholine and reduced tissue levels of ROS.

\section{Conflict of Interest \\ None declared}

\section{Funding Statement}

Authors declare that this study was self-funded.

\section{REFERENCES}

Ahmed, S., Tsukahara, S., Shwe, T.T.W., Yamamoto, S., Kunugita, N., Arashidani, K., et al. (2007) Effects of low-level formaldehyde exposure on synaptic plasticity-related gene expression in the hippocampus of immunized mice. Journal of Neuroimmunology. 186(1-2):104-111. https://doi.org/ 10.1016/j/.jneuroim.2007.03.010.

Ahmed, S.A., Ali, F.B. and Alaa, N.S. (2018) Histological and histometrical characteristics of the vesicular gland in non-breeding rams. Journal of Entomology and Zoology Studies. 6(1):1218-1221.

Andersen, B.B. and Pakkenberg, B. (2003) Stereological quantitation in cerebellar of people with schizophrenia. British Journal of Psychiatry. 182(4):354-361. https://doi.org/10.1192/bjp.182.4.354 Andersen, J.K. (2004) Oxidative stress in neurodegeneration: cause or consequence. Nature Medicine. 10:S18-S25. https://doi.org/10.1038/nrn143 4.

Anderson, J. (1989) Histometry in Theory and Practice of Histological Techniques. 3rd ed. Churchill Livingstone: London. Pp. 345-346.

Brandies, R., Brandys, Y. and Yehuda, S. (1989) The use of Morris water maze in the study of memory and learning. International Journal of Neuroscience. 48(12):29-69.https://doi.org/10.3109/00207458909002151 Brown, R.E., Corey, S.C. and Moore, A.K. (1999) Differences in measures of exploration and fear in $\mathrm{MHC}-$ congenic $\mathrm{C} 57 \mathrm{BL} / 6 \mathrm{~J}$ and $\mathrm{B} 6-\mathrm{H}-2 \mathrm{~K}$ mice. Behavior Genetics, 26:263-271. https://doi.org/10.102 3/A:1021694307672.

David, R.M., Moore, M.R., Finney, D.C. and Guest, D. (2000) Chronic toxicity of di(2-ethylhexyl) 
phthalate in rats. Toxicological Sciences. 55(2):433443. https://doi.org/10.1093/toxsci/55.2.433.

DeZwart, L.L., Meerman, J.H., Commandeur, J.N. and Vermeulen, N.P. (1999) Biomarkers of free radical damage applications in experimental animals and in humans. Free Radical Biology and Medicine. 26(1-2):202-226. https://doi.org/10.1016/S0891-5849 (98)00196-8

Ding, R. and Yang, Y. (2010) Aluminum chloride induced oxidative damage on cells derived from hippocampus and cortex of ICR mice. Brain Research. 1324:96-102. https://doi.org/10.1016/j.brai nres.2010.02.024.

Drapeau, E., Mayo, W., Aurousseau, C., Moal, M., Piazza, P. and Abrous, D.N. (2003) Spatial memory performances of aged rats in water maze predict levels of hippocampal neurogenesis, Proceedings of the National Academy of Sciences of the United States of America. 100(24):14385-14390. https://doi.org/10.1073/pnas.2334169100.

Evison, B.J., Chiu, F., Pezzoni, G., Phillips, D.R. and Cutts, S.M. (2008) Formaldehyde-activated pixantrone ismono functional DNA alklator that binds selectively to CPG and CPA doublet. Molecular Pharmacology. 74(1):184-194. https://doi.org/10.1123 /mol.108.045625.

Gao, X., Lai, C., Scott, T., Shen, J., Cai, T., Ordovas, J.M., et al. (2010) Urinary 8-hydroxy-2deoxyguanosine and cognitive function in Puerto Rican adults. American Journal of Epidemiology. 172(3):271-278. https://doi.org/10.1093/aje/kwq136.

Gutteridge, J.M. (1995) Lipid peroxidation and antioxidants as biomarkers of tissue damage. Clinical Chemistry. 41(12):1819-1828. https://doi.org/10.109 3/clinchem/41.12.1819.

Hang, C., Yahui, K. and Hong, Z. (2011) Oxidative stress, mitochondrial dysfunction, and aging. Journal of Signal Transduction. 646354. https://doi.org/10. 1155/ 2012/646354.

Hegazy, A., Elsayed, N., Ahmed, M. and Mohammad, N. (2017) Effect of formaldehyde on rat testis Structure. Academia Anatomica International, 3(2):15-23. doi: 10.21276/aanat.2017.3.2.4

Huda, M.A. and Zaid, A.A. (2007) Photo-histometry a modified computer assisted Morphometric measurement program. Journal of Faculty of Medicine, Baghdad. 49(1):139-145. https://doi.org /10.32007/jfacmedbagdad.4911435

Institute of Laboratory Animal Resources Committee on care and use of laboratory animals (2011). Guide for the care and use of laboratory animals. National Academies Press: Washington D.C. Accessed from https://www.nap.edu/catalog/12910/guide-for-thecare-and-use-of-laboratory-animals-eighth.

Janero, D.R. (1990) Malondialdehyde and thiobarbituric acid-reactivity as diagnostic indexes of lipid-peroxidation and peroxidative tissue-injury. Free Radical Biology and Medicine. 9(6): 515-540. https://doi.org/10.1016/0891-5849(90)90131-2
Jelsing, J., Gundersen, H., Nielsen, R., Hemmingsen, R. and Pakkenberg, B. (2006) The postnatal development of cerebellar Purkinje cells in the Gottingen minipig estimated with a new stereological sampling technique- the vertical bar fractionators. Journal of Anatomy. 209(3):321-331. https://doi.org/10.1111/j.1469-7580.2006.00611.x

Kaizaki, A., Tien, L., Pang, Y., Cai, Z., Tanaka, S., Numazawa, S., et al. (2003) Celecoxib reduces brain doperminergic neuronal dysfuction and improves sensorimotor behavioural performance in neonatal rats exposed to lipopolysaccharide. Journal of Neuroinflamation. 10(45):1-14.

Kurata, M., Suzuki, M. and Agar, N.S. (1993) Antioxidant systems and erythrocyte life-span in mammals. Comparative Biochemistry and Physiology. 106(3):477-487. https://doi.org/10.1016/ 0305-0491(93)90121-K

Lai, P.C., Huang, Y.T., Wu, C.W., Lai, C., Wang, P.J. and Chiu, T.H. (2011) Ceftriaxone attenuates hypoxic-ischemic brain injury in neonatal rats. Journal of Biomedical Science, 18(69):1-10. https://doi.org/10.1186/1423-0127-18-69

Leger, M., Quiedeville, A., Bouet, V., Haelewyn, B., Boulouard, M., Schumann-Bard, P. and Freret, T. (2013) Object recognition test in mice, Nature Protocols. 8(12):2531-2537. doi:10.1038/nprot.s Liu, Y., Ye, Z., Yang, H., Zhou, L., Fan, D., He, S., and Chui, D. (2010) Disturbances of soluble Nethylmaleimide-sensitive factor attachment proteins in hippocampal synaptosomes contribute to cognitive impairment after repetitive formaldehyde inhalation in male rats. Neuroscience. 169(3):1248-1254. https://doi.org/10.1016/j.neuroscience.2010.05.061 Liu, Z., Niu, W., Yang, X. and Wang, Y. (2013) Effects of combined acupuncture and eugenol on learning-memory ability and antioxidation system of hippocampus in Alzheimer disease rats via olfactory system stimulation. Journal of Traditional Chinese Medicine. 33(3):399-402. https://doi.org/10.1016/S0254-6272(13)60186-7.

Lowry, O.H., Rosebrough, N.J., Farr A.L. and Randall, R.J. (1951) Protein measurement with the Folin-Phenol reagent. Journal of Biological Chemistry.193:265-275.

Lu, Z., Li, C.M., Qiao, Y., Yan, Y. and Yang, X. (2008) Effect of inhaled formaldehyde on learning and memory of mice. Indoor Air. 18(2):77-83. doi:10.1111/j.1600-0668.2008.00524.

Mahmoud, M.S and Marwa, M.A. (2017) Neuroprotective effects of eugenol against aluminium induced toxicity in the rat brain. Archives of Industrial Hygiene and Toxicology. 68(1):27-39. https://doi.org/10.1515/aiht-2017-68-2878

Malek, F.A., Moritz, K.U. and Fanghanel, J. (2003) A study on the effect of inhalative formaldehyde exposure on water labyrinth test performance in rats. Annals of Anatomy. 185(3):277-285. https://doi.org/10.1016/S0940-9602(03)80040-7. 
Mecocci, P., MacGarvey, U. and Beal, M.F. (1994) Oxidative damage to mitochondrial DNA is increased in Alzheimer's disease. Annals of Neurology. 36(5):747-751.https://doi.org/10.1002/ana.410360510 Mesole, S.B., Okpanachi, O.A., Yusuf, U.A., Lwiindi, L. and Ndhlovu, D. (2020) Apoptotic inducement of neuronal cells by aluminium chloride and the neuroprotective effect of eugenol in Wistar rats. Oxidative Medicine and Cellular Longetivity, 8425643. https://doi.org/10.1155/2020/8425643.

Miranda, M.I. and Bermúdez-Rattoni, F (1997) Acetylcholine determination of microdialysates of fetal neocortex grafts that induce recovery of learning. Brain Research Protocols. 2(3):215-222. https://doi.org/10.1016/S1385-299X(97)00046-9.

Morris, R. (1984) Development of a water maze procedure for studying spatial learning in the rat. Journal of Neuroscience Methods. 11(1):47-60. https://doi.org/10.1016/0165-0270(84)90007-4

Morris, R., Garrud, P., Rawlins, J. and O'Keefe, J. (1982) Place navigation impaired in rats with hippocampal lesions. Nature. 297:681-683. https://doi.org/10.1038/297681a0.

Nazaroff, W.W. and Singer, B.C. (2004) Inhalation of hazardous air pollutants from environmental tobacco smoke in US residences. Journal of Exposure Analysis and Environmental Epidemiology. 14:71-77. https://doi.org/10.1038/sj.jea.7500361

Nishigori, H., Yasunaga, M., Masashi, M. and Watsuru, M. (1989) Preventive effects of pyrroloquinoline quinone on formation of cataract and decline of lenticular and hepatic glutathione of developing chick embryo after glucocorticoid treatment. Life Sciences. 45(7):593-598. https://doi.org/10.1016/0024-3205(89)90044-1.

Pitten, F.A., Kramer, A., Herrmann, K., Bremer, J. and Koch, S. (2000) Formaldehyde neurotoxicity in animal experiments. Pathology Research and Practice. 196(3):193-198. https://doi.org/10.1016/ S0344 -0338(00)80100-4.
Podhorna, J. and Brown, R.E. (2004) Strain differences in activity and emotionality do not account for differences in learning and memory performance between C57BL/6 and DBA/2 mice. Genes, Brain and Behavior. 1(2):96-110. https://doi.org/10.1034/j. 1601-183X.2002.10205.x

Ridley, R.M., Bowes, P.M. Baker, H.F. and Crow, T.J. (1984) An involvement of acetylcholine in object discrimination learning and memory in the marmoset. Neuropsycologia 22(3):53-263. Doi: 10.1016/00283932(84)90073-3.

Shigenaga, M.K., Hagen, T.M., Ames, B.N. (1994) Oxidative damage and mitochondrial decay in aging. Proceedings of the National Academy of Sciences of the United States of America. 91(23):10771-10778. https://doi.org/10.1073/pnas.91.23.10771

Sies, H. (1991) Oxidative stress: from basic research to clinical application. American Journal of Medicine. 91(3):31-38. https://doi.org/10.1016/0002-9343(91)9 0281-2

Thaysen-Andersen, M., Jørgensen, S.B., Wilhelmsem, E.S., Petersen, J.W. and Højrup, P. (2007) Investigation of the detoxification mechanism of formaldehyde-treated tetanus toxin. Vaccine. 225(12) 2213-2227. https://doi.org/10.1016/j.vaccine.2006.12. 033.

Til, H.P., Woutersen, R.A., Feron. V.J. and Clary, J.J. (1988) Evaluation of the oral toxicity of acetaldehyde and formaldehyde in a 4-week drinking-water study in rat. Food and Chemical Toxicology, 26(5):447-452. doi:10.1016/0278-6915(88)90056-7.

Yang, X., Huang, W., Wen, J. and Lin, T. (2007) Formaldehyde content in dried edible fungi from Dongguan market. Chinese Journal of Food Hygiene. 19(2):150-152.

Zendehdel, R., Fazli Z. and Mazinani, M. (2016) Neurotoxicity effect of formaldehyde on occupational exposure and influence of individual susceptibility to some metabolism parameters. Environmental Monitoring and Assessment. 188(11):648. doi:10.1007/s10661-016-5662-z.

Cite as Mesole, S.B., Okpanachi, O.A., Adebisi, S.S., Yusuf, U.A., Godam, E.T., Chisunka, C.G., Ivang, A., Abdulrazaq, A.A. and Kolawole, T.J. (2020) Evaluation of the neuroprotective effects of eugenol on formaldehyde induced neurotoxicity in Wistar rats. Nig. J. Neurosci. 11(2):62-70. http://doi.org/10.47081/njn2020.11.2/002 